Matriks Sains Matematik (MSMK)

DOI : http://doi.org/10.26480/msmk.02.2018.01.06

ZIBELLINE INTER RNATIONAL

ISSN: 2521-0831 (Print)

ISSN: 2521-084X (Online)

CODEN : MSMADH

\title{
STABILITY ANALYSIS OF PERIODIC AND ALMOST-PERIODIC DISCRETE SWITCHED LINEAR SYSTEM
}

\author{
Akbar Zada, Sartaj Ali* \\ Department of Mathematics, University of Peshawar, Peshawar 25000, Pakistan \\ *Corresponding Author email: sartajalistd@uop.edu.pk
}

This is an open access article distributed under the Creative Commons Attribution License, which permits unrestricted use, distribution, and reproduction in any medium, provided the original work is properly cited.

\section{ARTICLE DETAILS}

\section{Article History:}

Received 12 November 2017

Accepted 12 December 2017

Available online 1 January 2018

\section{ABSTRACT}

This article shows a connection between the boundedness and uniform exponential stability of linear discrete switched system in the space of periodic and almost-periodic sequences. Comprehensively, we prove that a linear discrete switched system is uniformly exponentially stable if and only if the Cauchy problem has bounded solution.

\section{KEYWORDS}

Discrete Switched Linear Systems, Uniform Exponential Stability, Discrete Evolution Family, Periodic and AlmostPeriodic Sequences.

\section{INTRODUCTION}

Wetlands The switched systems are the hybrid dynamical systems which has a several numbers of discrete and continuous time subsystems and a technique that defines the switching rule between them, for detail see monographs such as in many research paper [1-3]. In fact, all the switched systems have many uses in everyday domestic and industrial utilities, i.e. lighting, personal computers, power plants, transportation vehicles and so on. Efficient operations of all such applications depend on the essential hidden work done by switched systems, whose behavior is determined by a suitable interconnection and control of analogue and digital devices [4].

In recent years, many researchers were studying dynamic behaviors of linear switched systems and most of them focused on stability, stabilization and controllability of such systems, for detail study with different approaches [5-7]. The stability of linear and nonlinear switched systems is divided into two main types, which are, the stability under arbitrary switching and the stability under constrained switching [8]. The concern with stability analysis problems under arbitrary switching, for which it must be necessary that all the subsystems are asymptotically stable. When all the subsystems are exponentially stable then it is easy to construct a divergent trajectory from any initial state for such switched system. Thus, speaking generally, the assumptions for subsystems stability is not sufficient to assure the stability of switched systems under arbitrary switching, except regarding some special cases [9-17].

The monograph shows a linear discrete switched system:

$$
\beta(p+1)=\mathbf{H}_{\delta(p)} \beta(p)
$$

is uniformly exponentially stable if and only if a solution of the following problem:

$$
\left\{\begin{array}{l}
\beta(p+1)-\mathbf{H}_{\delta(p)} \beta(p)+e^{i \gamma(p+1)} \mathbf{M}(p+1), p \in \mathbf{Z}_{+},\left(\mathrm{T}_{\delta(p)}, \mathbf{M}\right) \\
\beta(0)=0,
\end{array}\right.
$$

is bounded, where $\mathbf{M}(p)$ with $\mathbf{M}(0)=0 \in \mathrm{AP}_{1}\left(Z_{+}, X\right)$,

$\delta:[0,1,2,3, \ldots) \rightarrow \mathrm{R}_{+}$with $\delta(1)=1$, is an arbitrary switching signals, $\mathbf{H}_{\delta(p)}$ is a sequence of the discrete transition matrices and $\operatorname{AP}_{1}\left(Z_{+}, X\right)$ is an almost-periodic sequences space, for detail study of almost-periodic spaces [18]. The remaining sections of this monograph are arranged as follow. Section 2 contains some basic facts, assumptions regarding $\operatorname{AP}_{1}\left(Z_{+}, X\right)$ and few auxiliary lemma's, which is devoted to uniform exponential stability concept. Section 3 contain a result that shows a uniform stability of exponential type for a linear periodic discrete switched system in way of showing that a solution of their corresponding Cauchy problem is bounded. Section 4 is devoted to the study of uniform stability of exponential type regarding linear almostperiodic discrete switched system.

\section{NOTATION AND PRELIMINARIES}

Consider a Banach space $X$ with a norm \|.\|. The notations $\mathrm{R}, \mathrm{R}_{+}$, $\mathbf{Z}$ and $Z_{+}$denotes the sets of real numbers, non-negative real numbers, integers and non-negative integers, respectively. Assume that a space of all $X$-valued sequences which is bounded, represented by 
$\mathbf{B}\left(Z_{+}, X\right)$, endowed with a norm which is considering to be supremum. Further, the notion $\mathrm{P}^{\mathrm{d}}\left(Z_{+}, X\right)$ denote a space of $\mathbf{d}$-periodic sequences, where $\mathbf{d} \geq 2$. From above discussion we clearly see that the space $\mathrm{P}^{\mathrm{d}}\left(Z_{+}, X\right)$ is a closed subspace of $\mathbf{B}\left(Z_{+}, X\right)$.

Next, we consider an operator $\tau=\xi_{\delta}(\mathbf{d}, 0)$, which is taken to be bounded and linear. The spectrum of $\tau$ is denoted by $\rho(\tau)$ and its spectral radius by $\mathbf{R}(\tau)$, which is defined as $\mathbf{R}(\tau)=\sup \{|\alpha|: \alpha \in \rho(\tau)\}$. From literature we is know that $\mathbf{R}(\tau)=\lim _{n \rightarrow \infty}\left\|\tau^{n}\right\|^{\frac{1}{n}}$ and $\varsigma(\tau)=\mathrm{C} \backslash \rho(\tau)$, where $\varsigma(\tau)$ is the resolving set of $\tau$.

Further, we are aware of the fact that a solution of a linear discrete switched system $\beta(p+1)=\mathbf{H}_{\delta(p)} \beta(p)$ or its corresponding Cauchy problem $\beta(p+1)=\mathbf{H}_{\delta(p)} \beta(p)+e^{i \gamma(p+1)} \mathbf{M}(p+1), p \in \mathbf{Z}_{+}$,

tends toward the idea of their discrete transition matrix. More detail explanations are neglected due to the sake of brevity but to make this monograph self-dependent, we give some definitions and lemmas on the basis of which we can prove our main result.

Definition 2.1. For each $n \in Z_{+}$, if we can find a constant $\lambda>0$ such that $\left\|\tau^{n}\right\| \leq \lambda$, then $\tau$ is said to be power bounded.

Definition 2.2. The family $\Gamma=\left\{\xi_{\delta}(p, q): p \geq q \geq 0\right\}$ of bounded linear operators, called $\mathbf{d}$-periodic for $\mathbf{d} \geq 2$, if $\Gamma$ satisfies the below conditions:

- $\xi_{\delta}(p, p)=\mathbf{I}_{1}$, for all $p \in \mathbf{Z}_{+}$, where $\mathbf{I}_{1}$ denotes the identity operator.

- $\xi_{\delta}(p, q) \xi_{\delta}(q, \boldsymbol{r})=\xi_{\delta}(p, \boldsymbol{r})$, for all $p \geq q \geq \mathbf{r}$ and $p, q, \mathbf{r} \in \mathbf{Z}_{+}$.

- $\xi_{\delta}(p+\mathrm{d}, q+\mathrm{d})=\xi_{\delta}(p, q)$, for all $p \geq q$ and $p, q \in \mathbf{Z}_{+}$

Lemma 2.3. For any $\mathbf{M}(p) \in \mathrm{AP}_{1}\left(Z_{+}, X\right)$ with $\mathbf{M}(0)=0$, the problem

$$
\left\{\begin{array}{l}
\beta(p+1)-\mathbf{H}_{\delta(p)} \beta(p)+e^{i \gamma(p+1)} \mathbf{M}(p+1), p \in \mathbf{Z}_{+},\left(\mathrm{T}_{\delta(p)}, \mathbf{M}\right) \\
\beta(0)=0,
\end{array}\right.
$$

has a solution

$$
\beta(p)=\sum_{k=1}^{p} \xi_{\delta}(p, k) e^{i \gamma k} \mathbf{M}(k),
$$

where $\delta:[0,1,2,3, \ldots) \rightarrow \mathrm{R}_{+}$with $\delta(1)=1$, is an arbitrary switching signals and $\mathbf{H}_{\delta(p)}$ is a sequence of the discrete transition matrices.

The proof of above lemma is so simple that's why we omit that proof. Assume that $M$ is any square matrix having order equal to one or greater than one and its spectrum is denoted by $S$, then we state the following lemma's as follow.

Lemma 2.4. Consider $\left\|M^{n}\right\|<\infty$, for all $n \in \mathrm{Z}_{+}$, then $|\alpha| \leq 1$, for each $\alpha \in S$.

Proof. If we consider that $|\alpha|>1$, then we can find a non-zero vector $v$ such that $M \alpha=\alpha v$, from this we can write that $M^{n} v=\alpha^{n} v$, for all $n \in Z_{+}$and thus $\left\|M^{n}\right\| \geq \frac{\left\|M^{n} v\right\|}{\|v\|}=|\alpha|^{n} \rightarrow \infty$ when $n \rightarrow \infty$. Hence $|\alpha| \leq 1$.

Lemma 2.5. Assume that $\left\|\sum_{k=0}^{m} M^{k}\right\|<\infty$ for each $m \in Z_{+}$, then 1 is not in the spectrum of $M$, i.e. $1 \notin S$.

Proof. On contrary let $1 \in S$ then $M q=q$ for some non-zero vector $q$ and $M^{n} q=q$, for all $n=1,2,3, \ldots, m$. Therefore,

$$
\begin{aligned}
\sup _{m \in Z_{+}}\left\|\sum_{k=0}^{m} M^{k}\right\| & =\sup _{m \in Z_{+}} \sup _{q \neq 0} \frac{\left\|\left(1+M+\ldots+M^{m}\right)(\theta)\right\|}{\|\theta\|} \\
& \geq \sup _{m \in Z_{+}} \frac{\|q\|}{q}=\infty,
\end{aligned}
$$

hence, we get that 1 is not in the spectrum of $M$, i.e. $1 \notin S$.

Corollary 2.6. If $\left\|\sum_{j=o}^{l}\left(e^{i v} \tau\right)^{j}\right\|<\infty$, for each $v \in \mathrm{R}$ and $m \in \mathrm{Z}_{+}$, then we have $e^{-i v} \notin \mathbf{R}(\tau)$.

Proof. Let us consider that $M=e^{i v} \tau$, from Lemma 2.5, as we know that $1 \notin \mathbf{R}\left(e^{i v} \tau\right)$, so $I-e^{i v} \tau$ is clearly an invertible matrix, which implies that $e^{-i \beta} \notin \mathbf{R}(\tau)$.

Lemma 2.7. Consider an operator $\tau$, which is bounded and linear with

$$
\operatorname{supsup}_{\gamma \in \mathbb{R}} \sum_{n \in \mathbf{Z}_{+}}^{n} e_{u=0}^{i \gamma k} \tau^{u}<\infty,
$$

then $\mathbf{R}(\tau)<1$, which means that $|\alpha| \leq 1$, for any $\alpha \in \rho(\tau)$. Proof. As we have

$$
\begin{gathered}
I_{1}-\left(e^{i \gamma} \tau\right)^{m}=I_{1}-\left(e^{i \gamma} \tau\right)\left(I_{1}+e^{i \gamma} \tau+\ldots+\left(e^{i \gamma} \tau\right)^{m-1}\right), \text { for each } \\
m \in \mathrm{Z}_{+} \text {and } \gamma \in \mathrm{R}_{+} .
\end{gathered}
$$

By applying norm to both sides of the above equation, we can get

$$
\begin{aligned}
\left\|I_{1}-\left(e^{i \gamma} \tau\right)^{m}\right\| & \leq 1+\left\|I_{1}-\left(e^{i \gamma} \tau\right)\right\|\left\|\left(I_{1}+e^{i \gamma} \tau+\ldots+\left(e^{i \gamma} \tau\right)^{m-1}\right)\right\| \\
& \leq 1+(1+\|\tau\|) \lambda
\end{aligned}
$$

By Lemma 2.4, we can write $|\alpha| \leq 1$, for each $\alpha \in S$ and using Lemma 2.5, we know that $e^{-i \gamma} \in \mathbf{R}(\tau)$, which implies that for any $\alpha \in \rho(\tau)$ and $|\alpha|<1$, hence we get $\mathbf{R}(\tau)<1$.

Definition 2.8. For any two constants $\aleph_{0}>0$ and $\wp>0$, if we have:

$$
\left\|\xi_{\delta}(p, q)\right\| \leq \aleph_{0} e^{\wp(p-q)} \text { for all } p \geq q \in \mathrm{Z}_{+},
$$

then $\Gamma$ is said to be bounded exponentially.

Definition 2.9. The growth bound of $\Gamma$ is denoted by $\mathbf{G}(\Gamma)$ and is defined as:

For each $\wp$ in $R$ and $\aleph \geq 0$, if the Eq. (2.1) is satisfied, then the smallest value in all $\wp \in R$ is said to be the growth bound of $\Gamma$. As we know

$$
\mathbf{G}(\Gamma)=\lim _{p \rightarrow \infty} \frac{\ln \left\|\xi_{\delta}(p, 0)\right\|}{p}=\frac{1}{\mathrm{~d}} \ln (\mathbf{R}(\tau)) .
$$

In detail, we can show the above equality as follow

$$
\begin{aligned}
\mathbf{G}(\Gamma) & =\lim _{p \rightarrow \infty} \frac{\ln \left\|\xi_{\delta}(p, 0)\right\|}{p} \\
& =\lim _{p \rightarrow \infty} \frac{\ln \left\|\xi_{\delta}(u \mathbf{d}, 0)\right\|}{u \mathbf{d}} \\
& =\frac{1}{\mathbf{d}} \lim _{p \rightarrow \infty} \ln \left\|\xi_{\delta}^{p}(\mathbf{d}, 0)\right\|^{\frac{1}{p}} \\
& =\frac{1}{\mathbf{d}} \ln \lim _{p \rightarrow \infty}\left\|\xi_{\delta}^{p}(\mathbf{d}, 0)\right\|^{\frac{1}{p}} \\
& =\frac{1}{\mathbf{d}} \ln \left(\mathbf{R}\left(\xi_{\delta}(\mathbf{d}, 0)\right)\right) \\
& =\frac{1}{\mathbf{d}} \ln (\mathbf{R}(\tau)) .
\end{aligned}
$$

Summarizing, we can say that a family $\Gamma$ is uniformly stable of 
exponential type if $\mathbf{G}(\Gamma)$ is negative.

Lemma 2.10. Let $\Gamma=\left\{\xi_{\delta}(p, q): p, q \geq 0\right\}$ be acting on a Banach space $X$. The below mention statements are equivalent:

(1) $\xi_{\delta}(p, q)$ is exponentially uniform stable.

(2) For any two constants $\aleph_{0}>0$ and $\wp>0$, we have

$$
\left\|\xi_{\delta}(p, q)\right\| \leq \aleph_{0} e^{-\wp(p-q)} \text { for all } p \geq q \in \mathbf{Z}_{+} .
$$

(3) The value of spectral radius of $\xi_{\delta}(p, 0)$ is less than one, i.e.

$$
\begin{aligned}
\mathbf{R}\left(\xi_{\delta}(p, 0)\right) & =\sup \left\{|\beta|: \beta \in \rho\left(\xi_{\delta}(p, 0)\right)\right\} \\
& =\lim _{u \rightarrow \infty}\left\|\xi_{\delta}(p)^{u}\right\|^{\frac{1}{k}}<1 .
\end{aligned}
$$

(4) For any $\alpha \in \mathrm{R}$, we have

$$
\sup _{p \geq 1}\left\|\sum_{k=1}^{p} e^{-i \eta k} \xi_{\delta}(p, k)^{k}\right\|=\aleph_{0}(\alpha)<\infty .
$$

As the proof of $(1) \Rightarrow(2) \Rightarrow(3) \Rightarrow(4)$ is obvious from the definitions. The proof of (4) $\Rightarrow(1)$ is same as Lemma 1 of [19].

Consider the problem $\left(T_{\delta(p)}, \mathbf{M}\right)$, which has a solution in the following form:

$$
\beta(p)=\sum_{k=1}^{p} \xi_{\delta}(p, k) e^{i \gamma k} \mathbf{M}(k) .
$$

Now we divide $p$ in terms of $\mathbf{d}$ such that $p=l \mathbf{d}+\boldsymbol{r}$ for some $l \in Z_{+}$and $\mathbf{r} \in\{0,1, \ldots, \mathbf{d}-1\}$. We consider the following sets:

$$
\mathbf{A}_{m}=\{1+m \mathbf{d}, 2+m \mathbf{d}, \ldots,(m+1)(\mathbf{d}-1)\} \text { for all } m \in \mathbf{Z}_{+}
$$

Now when $r \in\{1,2, \ldots, \mathbf{d}-1\}$, then we write

$$
\begin{gathered}
\mathbf{A}_{l}=\{l \mathbf{d}+1, l \mathbf{d}+2, \ldots, l \mathbf{d}+\mathbf{r}\}, \\
\mathbf{A}_{\mathbf{d}}=\{0, \mathbf{d}, 2 \mathbf{d}, \ldots, l \mathbf{d}\}
\end{gathered}
$$

So, we have

$$
\bigcup_{m=0}^{l-1} \mathbf{A}_{m} \bigcup \mathbf{A}_{l} \bigcup \mathbf{A}_{\mathrm{d}}=\{0,1,2, \ldots, p\}
$$

thus, from partition given in Eq. (2.2), the space $\mathbf{T}$ is constructed in the following way

$$
\mathbf{M}(u)= \begin{cases}(u-m d)[(1+m) d-u] \xi_{\delta}(u-m d, 0), & \text { if } \quad u \in \mathrm{A}_{m} \\ u(d-u) \xi_{\delta}(u, 0), & \text { if } \quad u \in \mathrm{A}_{l} \\ 0, & \text { if } \quad u \in \mathrm{A}_{d}\end{cases}
$$

i.e.

$$
\mathbf{T}=\{\mathbf{M}(p): \mathbf{M}(p) \text { satisfies }(2.3)\} .
$$

Clearly, $\mathbf{T}$ is the subspace of $\mathbf{d}$-periodic space.

We state the following results, where the space is considered to be space of periodic sequences. These results will be utilizing in the last section of this monograph for obtaining the main result.

Lemma 2.11. Spectral radius of $\tau$ is less than one, i.e., $\mathbf{R}(\tau)<1$ if and only if $\Gamma$ is exponentially uniform stable.

\section{EXPONENTIAL UNIFORM STABILITY OF PERIODIC SWITCHED LINEAR SYSTEM}

Theorem 3.1. Assume that $\Gamma=\left\{\xi_{\delta}(p, q): p, q \in Z_{+}\right\}$is an evolution discrete family acting on $X$. For any $\gamma \in \mathbf{R}$ and $\mathbf{d}$-periodic sequence $\mathbf{M}(p)$, the solution of the problem $\left(T_{\delta(p)}, \mathbf{M}\right)$ is bounded if and only if the family $\Gamma$ is exponentially uniform stable.

Proof. (1). Let us consider that $\Gamma$ is exponentially uniform stable, then we need to show that the solution of $\left(T_{\delta(p)}, \mathbf{M}\right)$ is bounded [20-27]. As the solution of $\left(T_{\delta(p)}, \mathbf{M}\right)$ is given by

$$
\beta(p)=\sum_{u=1}^{p} \xi_{\delta}(p, u) e^{i \eta u} \mathbf{M}(u) .
$$

Applying norm, we have

$$
\begin{aligned}
\|\beta(p)\| & =\left\|\sum_{u=1}^{p} e^{i \eta u} \xi_{\delta}(p, u) \mathbf{M}(u)\right\| \\
& \leq \sum_{u=0}^{p}\left\|e^{i \eta u} \xi_{\delta}(p, u) \mathbf{M}(u)\right\| \\
& =\sum_{u=0}^{p}\left\|e^{i \gamma u}\right\|\left\|\xi_{\delta}(p, u)\right\| \mathbf{M}(u) \| .
\end{aligned}
$$

Let $\|\mathbf{M}(u)\|=P_{z}$, then

$$
\begin{aligned}
\|\beta(p)\| & =\sum_{u=0}^{p} \aleph_{0} e^{-\wp(p-u)} P_{z} \\
& =P_{z} \aleph_{0} e^{-\wp p} \sum_{u=0}^{p} e^{\aleph_{0} u} \\
& =P_{z} \aleph_{0} e^{-\wp p p}\left(\frac{1-e^{(p+1) \aleph_{0}}}{1-e^{\aleph_{0}}}\right) \\
& \leq P_{z} \aleph_{0} e^{-\wp p p} \\
& <\infty .
\end{aligned}
$$

Thus, the solution of $\left(T_{\delta(p)}, \mathbf{M}\right)$ is bounded.

(2). Consider that the solution of $\left(T_{\delta(p)}, \mathbf{M}\right)$ is bounded, then we need to show the $\Gamma$ is exponentially uniform stable. For this let $\mathbf{M}(p)$ be any periodic sequence. Then

$$
\mathbf{M}(u)=\left\{\begin{array}{lll}
(u-m d)[(1+m) d-u] \xi_{\delta}(u-m d, 0), & \text { if } & u \in \mathrm{A}_{m} \\
u(d-u) \xi_{\delta}(u, 0), & \text { if } & u \in \mathrm{A}_{l} \\
0, & \text { if } & u \in \mathrm{A}_{d}
\end{array}\right.
$$

As,

$$
\begin{aligned}
\beta(p)= & \sum_{u=1}^{p} e^{i \eta u} \xi_{\delta}(p, u) \mathbf{M}(u) \\
= & \sum_{u=1}^{l d+r} e^{i \eta u} \xi_{\delta}(l d+r, u) \mathbf{M}(u) \\
= & \sum_{u \in \bigcup_{m=0}^{l-1} \mathrm{~A}_{m} \cup \mathrm{A}_{l} \cup \mathrm{A}_{d}}^{l d+r} e^{i \eta u} \xi_{\delta}(l d+r, u) \mathbf{M}(u) \\
= & \sum_{u \in \bigcup_{m=0}^{l-1} \mathrm{~A}_{m} \cup \mathrm{A}_{l} \cup \mathrm{A}_{d}}^{l d+r} e^{i \eta u} \xi_{\delta}(l d+r, u) \mathbf{M}(u) \\
& +\sum_{u \in \mathrm{A}_{l}}^{l d+r} e^{i \eta u} \xi_{\delta}(l d+r, u) \mathbf{M}(u) \\
& +\sum_{u \in \mathrm{A}_{d}}^{l d+r} e^{i \eta u} \xi_{\delta}(l d+r, u) \mathbf{M}(u) \\
= & \sum_{m=0}^{l-1} \sum_{u=1+l d}^{d-1+m d} e^{i \eta u} \xi_{\delta}(p, u) \mathbf{M}(u) \\
& +\sum_{u=l d+1}^{l d+r} e^{i \eta u} \xi_{\delta}(p, u) \mathbf{M}(u) \\
& +\sum_{u \in \mathrm{A}_{d}} e^{i \eta u} \xi_{\delta}(l d+r, u) \mathbf{M}(u) .
\end{aligned}
$$


By virtue of the $d$-periodic sequence $\mathbf{M}(u)$, we can write

$$
\begin{aligned}
\beta(p)= & \sum_{u=1}^{p} e^{i m u} \xi_{\delta}(p, u) \mathbf{M}(u) \\
= & \left.\sum_{j=0}^{l-1} \sum_{u=1+m d}^{d-1+m d} e^{i \eta u} \xi_{\delta}(l d+r, u)(u-m d)-u\right] \xi_{\delta}(u-m d, 0) \\
& +\sum_{u=l d+1}^{l d+r} e^{i \eta u} \xi_{\delta}(l d+r, u) u(d-u) \xi_{\delta}(u, 0) \\
= & W_{1}+W_{2},
\end{aligned}
$$

where,

$$
\begin{aligned}
& \left.W_{1}=\sum_{j=0}^{l-1} \sum_{u=1+m d}^{d-1+m d} e^{i \eta u} \xi_{\delta}(l d+r, u)(u-m d)-u\right] \xi_{\delta}(u-m d, 0) \\
& W_{2}=\sum_{u=l d+1}^{l d+r} e^{i j u} \xi_{\delta}(l d+r, u) u(d-u) \xi_{\delta}(u, 0)
\end{aligned}
$$

Writing $W_{1}$ in the form

$$
\begin{aligned}
& \left.W_{1}=\sum_{j=0}^{l-1} \sum_{u=1+m d}^{d-1+m d} e^{i n u} \xi_{\delta}(1+m) d-u\right] \xi_{\delta}(u-m d, 0) \\
& =\sum_{j=0}^{l-1} \sum_{u=1+m d}^{d-1+m d}[(1+m) d-u] \xi_{\delta}(u, m d)
\end{aligned}
$$

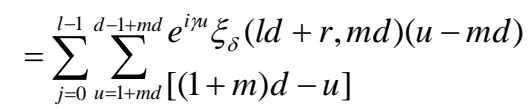

$$
\begin{aligned}
& =\sum_{j=0}^{l-1} \xi_{\delta}(l d+r, m d) \sum_{u=1+m d}^{d-1+m d}[(1+m) d-u] \\
& =\sum_{j=0}^{l-1} \xi_{\delta}(l d+r, m d) \sum_{u=1+m d}^{d-1+m d}[d-(u-m d)],
\end{aligned}
$$

which implies that

$$
\begin{aligned}
W_{1} & =\sum_{m=0}^{l-1} \xi_{\delta}(r, 0) \xi_{\delta}^{l-m}(d, 0) e^{i \gamma m d} \sum_{q=1}^{m-d} e^{i \gamma q} q(d-q) \\
= & \xi_{\delta}(r, 0) \sum_{q=1}^{d-1} e^{i \gamma q} q(d-q) \sum_{m=0}^{l-1} e^{i m m d} \xi_{\delta}^{l-m}(d, 0) \\
& =\xi_{\delta}(r, 0) \sum_{q=1}^{d-1} e^{i \gamma q} q(d-q) \sum_{\alpha=1}^{l} e^{i \gamma d(l-\alpha)} \xi_{\delta}^{\alpha}(d, 0) \\
& =\xi_{\delta}(r, 0) \sum_{q=1}^{d-1} e^{i \gamma q} q(d-q) e^{i \gamma d l} \sum_{\alpha=1}^{l} e^{-i \gamma d \alpha} \xi_{\delta}^{\alpha}(d, 0) \\
& =\mathbf{G}(r, d) \sum_{\alpha=1}^{l} e^{-i \gamma d \alpha} \xi_{\delta}^{\alpha}(d, 0)
\end{aligned}
$$

where $\mathbf{G}(\mathbf{r}, \mathbf{d})=\xi_{\delta}(\mathbf{r}, 0) \sum_{q=1}^{\mathrm{d}-1} e^{i \gamma q} q(\mathbf{d}-q) e^{i \gamma \mathrm{d} l} \neq 0$.

Furthermore,

$$
\begin{aligned}
W_{2} & =\sum_{u=l d+1}^{l d+r} e^{i \eta u} \xi_{\delta}(l d+r, u) u(d-u) \xi_{\delta}(u, 0) \\
& =\sum_{u=l d+1}^{l d+r} e^{i \eta u} \xi_{\delta}(l d+r, 0) u(d-u) \\
& =\xi_{\delta}(l d+r, 0) \sum_{u=l d+1}^{l d+r} e^{i \eta u} u(d-u) .
\end{aligned}
$$

Therefore,

$$
\begin{aligned}
\sum_{u=1}^{p} e^{i m u} \xi_{\delta}(p, u) \mathbf{M}(u)= & \mathbf{G}(\mathbf{r}, \mathbf{d}) \sum_{\alpha=1}^{l} e^{-i \gamma \mathbf{d} \alpha} \xi_{\delta}^{\alpha}(\mathbf{d}, 0) \\
& +\xi_{\delta}(l d+r, 0) \sum_{u=l d+1}^{l d+r} e^{i \eta u} u(d-u) .
\end{aligned}
$$

Since $\beta(p)$ is bounded, which implies that that $W_{1}$ is bounded, i.e.

$$
\operatorname{Sup}_{l \geq 0}\left\|\sum_{\alpha=1}^{l} e^{-i \gamma \mathbf{d} \alpha} \xi_{\delta}^{\alpha}(\mathbf{d}, 0)\right\|<\infty .
$$

Thus, with the help of Lemma 2.7, we realize that $\mathbf{R}\left(\xi_{\delta}(\mathbf{d}, 0)\right)<1$.

Finally, by Lemma 2.11, $\Gamma=\left\{\xi_{\delta}(p, q): p \geq q \geq 0\right\}$ is exponentially uniform stable.

\section{EXPONENTIAL UNIFORM STABILITY OF ALMOST PERIODIC SWITCHED LINEAR SYSTEM}

Here we take some assumptions which is helpful in proving our main result.

Now to obtain the main result, in this section we need to define a discrete transitions matrix acting on a space of $X$-valued sequences [28-31]. For such required aim, we must consider the below facts regarding some spaces.

The notion $\mathrm{B}\left(Z_{+}, X\right)$ represents a space of all $X$-valued bounded sequences, which must be uniformly convergent and its concern norm is defined as $\|\mathbf{M}\|_{\infty}=\sup _{p \in Z}\|\mathbf{M}(p)\|$. The notation $\mathbf{P}^{d}\left(Z_{+}, X\right)$ denotes a subspace of $\mathbf{B}\left(Z_{+}, X\right)$ containing $\mathbf{d}$-periodic sequences $\mathbf{F}$, which is almost-periodic such that $\mathbf{F}(p+\mathbf{d})=\mathbf{F}(p)$ for each $p \in Z$. Finally, the notation $\operatorname{AP}_{1}\left(Z_{+}, X\right)$ is devoted to a space of all $X$-valued sequences which is defined on $Z_{+}$in the following way:

$$
\mathbf{M}(p)=\sum_{u=-\infty}^{u=+\infty} e^{i \kappa_{u} p} C_{u}(\mathbf{M}),\|\mathbf{M}\|_{1}=\sum_{u=-\infty}^{u=+\infty}\left\|C_{u}(\mathbf{M})\right\|<\infty,
$$

where $p, \kappa_{u} \in \mathbf{Z}$ and $C_{u}(\mathbf{M}) \in X$.

Next, we consider that for any $p \geq 0$, the set $\hat{\mathbf{A}}_{p}$ contains all $X$ valued sequences which is defined on $Z$ such that we can find a sequence $\mathbf{F} \in \mathrm{P}^{d}\left(Z_{+}, X\right) \mathbf{A P}_{1}\left(Z_{+}, X\right)$ with $\mathbf{F}(p)=0$. Further, for any $q<p$ we have $\mathbf{M}=\mathbf{F}_{\mid\{p, p+1, \ldots\}}$ and $\mathbf{M}(q)=0$.

For obtaining our required result, we also consider that

$$
\hat{\mathbf{A}}_{p}=\left\{e^{i \kappa} \otimes \mathbf{M}: \kappa \in \mathrm{R} \text { and } \mathbf{M} \in \bigcup_{p \geq 0} \hat{\mathbf{A}}_{p}\right\}, \quad \mathrm{E}(\mathrm{Z}, X)=\operatorname{span}(\hat{\mathbf{A}})
$$

and a space $\tilde{\mathrm{E}}(Z, X)=\overline{\operatorname{span}}(\hat{\mathbf{A}})$ which represent a closed subspace of $\mathbf{B}\left(Z_{+}, X\right)$ and its concern norm is considered to be sup norm. The discrete transitions matrix $\eta=\left\{T_{\delta(p)}\right\}_{p \geq 0}$ with $\Gamma$ on $\tilde{\mathrm{E}}(Z, X)$ is define as:

$$
T_{\delta(q)} \dot{\mathbf{M}}(p)=\left\{\begin{array}{ccc}
\xi_{\delta}(p,(p-q) \dot{\mathbf{M}}(p-q), & \text { if } & p \geq q \\
0 & \text { if } & p<q,
\end{array}\right.
$$

for any $\dot{\mathbf{M}} \in \widetilde{\mathrm{E}}(Z, X)$.

Lemma The space $\tilde{\mathrm{E}}(Z, X)$, which is a closed subspace of $\mathbf{B}\left(Z_{+}, X\right)$, is invariant under the discrete transition matrix $T_{\delta(p)}$, defines in Eq. (3.1). Proof. Consider a sequence $\dot{\mathbf{M}}(p)$, a real number $\boldsymbol{\kappa}$, i.e. $\boldsymbol{\kappa} \in \mathrm{R}$ and $\dot{\mathbf{M}} \in \bigcup_{p \geq 0} \hat{\mathbf{A}}_{p}$. After that we can find $\mathbf{w} \geq 0$ and a sequence $\mathbf{F}(p) \in \mathbf{P}^{d}\left(\mathbf{Z}_{+}, X\right) \mathbf{A P}_{1}\left(Z_{+}, X\right)$ with $\mathbf{F}(\mathbf{w})=0, \mathbf{M}(p)=\mathbf{F}(p)$ for any $p \geq \mathbf{w}$ and $\mathbf{M}(p)=0$ for every $p<\mathbf{w}$. Hence, for each 


$$
\begin{aligned}
p \geq 0 & \text { and } q \in \mathbf{Z}, \text { we have } \\
T_{\delta(q)} \dot{\mathbf{M}}(p) & =\left\{\begin{array}{llc}
e^{i \kappa(p-q)} \xi_{\delta}(p-q) \mathbf{F}(p-q), & \text { if } & p \geq q+\mathbf{w} \\
0 & \text { if } & 0 \leq p<q+\mathbf{w}
\end{array}\right.
\end{aligned}
$$

The sequence $\mathbf{D}(p)=e^{-i \kappa(p-q)} \xi_{\delta}(p-q) \mathbf{F}(p-q)$ is $\mathbf{d}$-periodic such that

$\|\mathbf{D}().\| \leq\left\|\xi_{\delta}(p-q)\right\| \sum_{u=-\infty}^{\infty} e^{i n u(p-q)} C_{u}(\mathbf{F})\left\|\leq \zeta e^{\eta p}\right\| \mathbf{F}(.) \|_{1}<\infty^{\prime}$

for some $\zeta \geq 1$ and $\eta \in \mathrm{R}$, this implies that $T_{\delta(q)} \dot{\mathbf{M}} \in \hat{\mathbf{A}}$. Now as we have

$$
T_{\delta(q)} \dot{\mathbf{M}}=\alpha T_{\delta(q)} g+\beta T_{\delta(q)} h,
$$

where $\dot{\mathbf{M}}=\alpha g+\beta h \in \mathrm{E}(Z, X)$, with $g, h \in \hat{\mathbf{A}}$ and $\alpha, \beta$ are complex scalar. Thus $T_{\delta(p)}$ is a linear operator from $\tilde{\mathrm{E}}(Z, X)$ to $\mathrm{B}(\mathrm{Z}, X)$. But $T_{\delta(p)} g, T_{\delta(p)} h \in \hat{\mathbf{A}}$ and therefore $T_{\delta(p)} \dot{\mathbf{M}} \in \mathrm{E}(Z, X)$ . Hence, $\tilde{\mathrm{E}}(Z, X)$ is invariant under the discrete transition matrix $T_{\delta(p)}$.

Let $G=T_{\delta(1)}-I$ be a discrete transition matrix. For discrete transition matrix, the Taylor formula of order one is:

$$
T_{\delta(q)} \dot{\mathbf{M}}-\dot{\mathbf{M}}=\sum_{u=0}^{p-1} T_{\delta(u)} G \dot{\mathbf{M}}
$$

for each $1 \leq p \in \mathbf{Z}_{+}$and $\dot{\mathbf{M}} \in X$.

Lemma 4.2. For any $\dot{\mathbf{M}}, \mathbf{Y} \in \widetilde{\mathrm{E}}(Z, X)$. The below two statements are equivalent:

i. $\quad G \mathbf{Y}=-\dot{\mathbf{M}}$.

ii. $\mathbf{Y}(p)=\sum_{u=0}^{p} \xi_{\delta}(p, u) \dot{\mathbf{M}}(u)$, for any $u \in \mathbf{Z}_{+}$.

Proof. (i) $\Rightarrow$ (ii): With the help of Eq. (3.3), we can write:

$$
T_{\delta(q)} \mathbf{Y}-\mathbf{Y}=\sum_{u=0}^{p-1} T_{\delta(u)} G \mathbf{Y}-\sum_{u=0}^{p-1} T_{\delta(u)} G \dot{\mathbf{M}} .
$$

Thus, for any $p \in \mathbf{Z}_{+}$, we have

$$
\begin{aligned}
\mathbf{Y}(p) & =\left(T_{\delta(p)} \mathbf{Y}\right)(p)+\sum_{u=0}^{p-1} T_{\delta(u)} G \dot{\mathbf{M}}(p) \\
& =\xi_{\delta}(p, 0) y(0)+\sum_{u=0}^{p-1} \xi_{\delta}(p, p-u) \dot{\mathbf{M}}(p-u) \\
& =\sum_{u=0}^{p} \xi_{\delta}(p, u) \dot{\mathbf{M}}(u) .
\end{aligned}
$$

(ii) $\Rightarrow$ (i): As $G=T_{\delta(p)}-\mathbf{I}_{1}$, thus

$$
\begin{aligned}
G \mathbf{Y}(p) & =\left(T_{\delta(p)}-\mathbf{I}_{1}\right) \mathbf{Y}(p) \\
= & \left(T_{\delta(p)} \mathbf{Y}(p)-\mathbf{Y}(p)\right) \\
= & \xi_{\delta}(p, p-1) \sum_{u=0}^{p-1} \xi_{\delta}(p-1, u) \dot{\mathbf{M}}(u)-\mathbf{Y}(p) \\
= & \sum_{u=0}^{p-1} \xi_{\delta}(p, u) \dot{\mathbf{M}}(u)-\sum_{u=0}^{p} \xi_{\delta}(p, u) \dot{\mathbf{M}}(u) \\
= & -\dot{\mathbf{M}}(p) .
\end{aligned}
$$

Now we are in position to state and prove the main result of this monograph, which is stated as follow.

Theorem 4.3. If $W$ is v-periodic evolution discrete family acting on $X$ and $\eta$ is associated discrete transition matrix on $\widetilde{\mathrm{E}}(\mathrm{Z}, X)$, then the below statements are equivalent:

(1): $\Gamma$ is exponentially uniform stable.

(2): $\eta$ is exponentially uniform stable.
(3): G has an inverse.

(4): For any $\dot{\mathrm{M}} \in \tilde{\mathrm{E}}(\mathrm{Z}, X)$, we have $\sum_{u=0}^{p} \xi_{\delta}(p-u, 0) \dot{\mathbf{M}}(u) \in \tilde{\mathrm{E}}(Z, X)$.

(5): For all $\dot{\mathbf{M}} \in \mathrm{P}^{d}(\mathrm{Z}, X), \quad \sum_{u=0}^{p} \xi_{\delta}(p-u, 0) \dot{\mathbf{M}}(u)$ is bounded on $\mathrm{Z}_{+}$.

Proof. $\quad(1) \Rightarrow(2): \quad$ Consider $\quad \Gamma=\left\{\xi_{\delta}(p, q): p \geq q \geq 0\right\} \quad$ is exponentially uniform stable, then by definition, we can find two constants $\aleph_{0}>0$ and $\wp>0$, which satisfies the below inequality:

$$
\left\|\xi_{\delta}(p, q)\right\| \leq \aleph_{0} e^{-\wp(p-q)} \text { for all } p \geq q \geq \in Z_{+}
$$

Now for all $\dot{\mathbf{M}} \in \widetilde{\mathrm{E}}(Z, X)$ and $p \geq 0$, the norm is definition as:

$$
\begin{aligned}
\left\|T_{\delta(q)} \dot{\mathbf{M}}\right\|_{\tilde{\mathrm{E}}(Z, X)} & =\sup _{p \geq q}\left\|\xi_{\delta}(p-q, 0) \dot{\mathbf{M}}(p-q)\right\| \\
& \leq \aleph_{0} e^{-\wp(p-q)} \sup _{p \geq q}\|\dot{\mathbf{M}}(p-q)\| \\
& =\aleph_{0} e^{-\wp(p-q)}\|\dot{\mathbf{M}}\|_{\tilde{\mathrm{E}}(Z, X)} .
\end{aligned}
$$

As $\dot{\mathbf{M}} \in \tilde{\mathrm{E}}(Z, X)$, implies that $\dot{\mathbf{M}}$ is bounded. Thus $\left\|T_{\delta(q)} \dot{\mathbf{M}}\right\|_{\tilde{\mathrm{E}}(Z, X)}<\infty$, which implies that $\eta$ is bounded, hence $\eta$ is uniformly exponentially stable.

(2) $\Rightarrow$ (3): W known that the evolution discrete family $\eta$ is exponentially uniform stable, if and only if $\mathbf{R}\left(T_{\delta(p)}\right)<1$, implies that $1 \notin \rho\left(T_{\delta(p)}\right)$. Hence $1 \in \zeta\left(T_{\delta(p)}\right)$ which implies that $G=T_{\delta(p)}-I$ has an inverse.

(3) $\Rightarrow$ (4): Let us consider that $G$ has an inverse, therefore for any $\dot{\mathbf{M}} \in \tilde{\mathrm{E}}(Z, X)$ we can find $\mathbf{Y} \in \tilde{\mathrm{E}}(Z, X)$ such that $G \mathbf{Y}=-\dot{\mathbf{M}}$. Hence by using Lemma 3.2 we obtain that

$$
\mathbf{Y}(p)=\sum_{u=0}^{p} \xi_{\delta}(p-u, 0) \dot{\mathbf{M}}(u)
$$

and in view of Lemma 3.1, we have

$$
\mathbf{Y}(p)=\sum_{u=0}^{p} \xi_{\delta}(p-u, 0) \dot{\mathbf{M}}(u) \in \tilde{\mathrm{E}}(Z, X) .
$$

(4) $\Rightarrow$ (5): As we know that $\mathbf{Y}(p)=\sum_{u=0}^{p} \xi_{\delta}(p-u, 0) \dot{\mathbf{M}}(u) \in \tilde{\mathrm{E}}(Z, X)$, which means that $\mathbf{Y}(p)=\sum_{u=0}^{p} \xi_{\delta}(p-u, 0) \dot{\mathbf{M}}(u)$ is bounded due to fact that $\tilde{\mathrm{E}}(Z, X)$ is a subset of $\mathbf{B}(Z, X)$.

(5) $\Rightarrow$ (1): It can be seen as direct outcome of Lemma 2.11 . From the above theorem, we have:

Corollary 4.4. The family of linear bounded operators $\Gamma$ is uniformly exponentially stable if and only if for each $\dot{\mathrm{M}} \in \widetilde{\mathrm{E}}(Z, X)$, the solution of the problem

$$
\left\{\begin{aligned}
\beta(p+1) & =\mathbf{H}_{\delta(p)} \beta(p)+\dot{\mathbf{M}}(p+1), \quad p \in \mathbf{Z}_{+} \\
\beta(0) & =0,
\end{aligned}\right.
$$

is bounded on $Z_{+}$.

\section{CONCLUSION}

On concluding note, in this paper we established a connection which reveals that a periodic and almost-periodic linear discrete switched system $\beta(p+1)=\mathbf{H}_{\delta(p)} \beta(p)$ is exponentially uniform stability if and only if the solution of their corresponding Cauchy problem $\left(T_{\delta(p)}, \mathbf{M}\right)$ is bounded. 


\section{COMPETING INTEREST}

Authors declare that they have no competing interest regarding this research work

\section{REFERENCES}

[1] DeCarlo, R., Branicky, M., Pettersson, S., Lennartson, B. 2000. Perspectives and results on the stability and stabilizability of hybrid systems. Proceedings of the IEEE, 1069-1082.

[2] Pettersson, S., Lennartson, B. 1997. Lmi for stability and robustness of hybrid systems. Proceedings of the American Control Conference, 1714-1718.

[3] Ye, H., Michel, A.N., Hou, L. 1996. Stability analysis of switched systems. Proceedings of the Conference on Decision and Control.

[4] Vasca, F., and Iannelli, L. 2012. Dynamics and control of switched electronic systems. London: Springer-Verlag.

[5] Cheng, D. 2000. Stabilization of planar switching systems. Systems \& Control Letters, 51 (2), 79-88.

[6] Ezzine, J., and Haddad, A.H. 1989. Controllability and observability of hybrid systems. International Journal of Control, 49 (6), 2045-2055.

[7] Xie, G., Wang, L. 2003. Controllability and stabilizable of switched linear systems. Systems \& Control Letters, 48 (2), 135-155.

[8] Liberzon, D. 2003. Switching in systems and control. Systems and Control: Foundations and Applications, Birkhauser Boston Inc., Boston, MA.

[9] Agrachev, A.A., Liberzon, D. 2001. Lie-algebra stability criteria for switched systems. SIAM Journal on Control and Optimization, 40 (1), 253-270.

[10] Asarin, E., Bournez, O., Dang, T., Maler, O., Pnueli, A. 2000. Effective synthesis of switching controllers for linear systems, in Proceeding of the IEEE: Special Issue on Hybrid Systems, P. J. Antsaklis and editor, Eds. New York: IEEE Press, 88, 1011-1025.

[11] Ji, Z., Feng, G., Guo, X. 2007. A constructive approach to reachability realization of discrete-time switched linear systems. Systems \& Control Letters, 56 (11-12), 669-677.

[12] Sun, Z., Ge, S.S. 2005. Switched Linear Systems: Control and Design, New York: Springer-Verlag.

[13] Sun, Z., Ge, S.S. 2011. Stability theory of switched dynamical systems. London: Springer-Verlag.

[14] Sun, Z., Ge, S.S., Lee, T.H. 2002. Controllability and reachability criteria for switched linear systems. Automatica, 38 (5), 775-786.

[15] Zada, A., Ali, S., Li, Y. 2017. Ulam-type stability for a class of implicit fractional differential equations with non-instantaneous integral imuplses and boundary condition. Advances in Difference Equations, $317,1-26$.

[16] Zhai, G., Hu, B., Yasuda, K., Michel, A.N. 2002. Qualitative analysis of discrete-time switched systems. Proceeding American Control Conference, 1880-1885.

[17] Zhai, G., Xu, X., Lin, H., Michel, A.N. 2006. Analysis and design of switched normal systems. Nonlinear Analysis, Theory, Methods \& Applications, 65, 2248-2259.

[18] Corduneanu, C. 2009. Almost Periodic Oscillations and Waves. Springer, NewYork, NY, USA.

[19] Buse, C., Cerone, P., Dragomir, S.S., Sofo, A. 2005. Uniform stability of periodic discrete system in Banach spaces. Journal of Difference Equations and Applications, 11 (12), 1081-1088.

[20] Bemporad, A., Ferrari-Trecate, G., Morari, M. 2000. Observability and controllability of piecewise affine and hybrid systems. IEEE Xplore: IEEE Transactions on Automatic Control, 45 (10), 1864-1876.
[21] Besicovitch, A.S. 1955. Almost Periodic Functions, Dover Publications Inc. New York, NY, USA.

[22] Buse, C., Zada, A. 2010. Dichotomy and boundedness of solutions for some discrete Cauchy Problems. Operator Theory Advances and Applications, 203, 165-174.

[23] Cheng, D. 2005. Controllability of switched bilinear systems. IEEE Xplore: IEEE Transactions on Automatic Control, 50 (4), 511-515.

[24] Ge, Y., Tian, L., Liu, Z. 2007. Survey on the stability of networked control systems. Control Theory and Technology, 5 (4), 374-379.

[25] Gurvits, L. 1995. Stability of discrete linear inclusion. Linear Algebra and its Applications, 231, 47-85.

[26] Hespanha, J.P., Liberzon, D., Angeli, D., Sontag, E.D. 2005. Nonlinear norm-observability notions and stability of switched systems. IEEE Xplore: IEEE Transactions on Automatic Control, 50 (2), 154-168.

[27] Mori, Y., Mori, T., Kuroe, Y. 1997. A solution of the common Lyapunov function problem for continuous-time systems. In Proceeding 36th Conference Decision and Control, 3530-3531.

[28] Narendra, K.S., Balakrishnan, J. 1994. A common Lyapunov function for stable LTI systems with commuting A-matrices. IEEE Xplore: IEEE Transactions on Automatic Control, 39, 2469-2471.

[29] Peleties, P., Decarlo, R. 1991. Asymptotic stability of m-switched systems using Lyapunov-like functions. In Proceeding of the 1991 American Control Conference, 1679-1684.

[30] Wicks, M.A., Peleties, P., DeCarlo, R. 1994. Construction of piecewise Lyapunov functions for stabilizing switched systems. In Proceeding of the 33rd IEEE Conference on Decision and Control, 3492-3497.

[31] Zhang, D., Yu, L., Wang, Q., Ong, C. 2013. Estimator design for discrete time switched neural networks with asynchronous switching and time varying delay. IEEE Transactions on Neural Networks and Learning Systems, 24, 1906-1906.

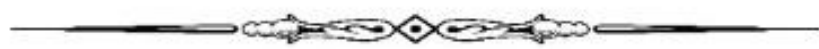

